\title{
Through-and-through Nasal Reconstruction with the Bi-Pedicled Forehead Flap
}

\author{
Tommaso Agostini, Raffaella Perello, Giulia Lo Russo, Giuseppe Spinelli \\ Department of Plastic and Reconstructive Surgery, University of Florence, Faculty of Medicine and Surgery, Florence, Italy
}

Background Nasal reconstruction is one of the most difficult challenges for the head and neck surgeon, especially in the case of complex full thickness defects following malignant skin tumor resection. Full-thickness defects require demanding multi-step reconstruction.

Methods Seven patients underwent surgical reconstruction of full-thickness nasal defects with a bi-pedicled forehead flap shaped appropriately to the defect. Patients were aged between 58 and 86 years, with a mean age of 63.4 years. All of the tumors were excised using traditional surgery, and in 4 of the patients, reconstruction was performed simultaneously following negativity of fresh frozen sections of the margins under general anesthesia.

Results Nasal reconstruction was well accepted by all of the patients suffering non-melanoma skin tumors with acceptable cosmetic outcomes. The heart-shaped forehead flap was harvested in cases of subtotal involvement of the nasal pyramid, while smaller defects were reconstructed with a wing-shaped flap. No cartilaginous or osseous support was necessary.

Conclusions This bi-pedicled forehead flap was a valid, versatile, and easy-to-implement alternative to microsurgery or multi-step reconstruction. The flap is the best indication for fullthickness nasal defects but can also be indicated for other complex facial defects in the orbital (exenteratio orbitae), zygomatic, and cheek area, for which the availability of a flap equipped with two thick and hairless lobes can be a valuable resource.

Keywords Nose neoplasms / Nose deformities, acquired / Surgical flaps / Nasal surgical procedures / Nasal bone
Correspondence: Tommaso Agostini Department of Plastic and Reconstructive Surgery, University of Florence, Largo Palagi 1, Florence, Italy

Tel: +39-050-996894

Fax: +39-0573-364760

E-mail: tommasoagostini@ymail.com

\section{INTRODUCTION}

The earliest examples of plastic surgery involved nasal reconstruction in India (2500-3000 BCE), in a society where nose and ear amputation was the most frequent punishment inflicted on enemies and unfaithful wives [1-3]. The earliest rhinoplasties were performed using local flaps taken from the cheek by Sushruta (600 BCE) [4]. Only later was nasal reconstruction attempted using local flaps from the forehead, a technique known nowadays as the Indian method. Guidelines for this approach were passed on for years until 1794 when a nasal reconstruction using a median forehead flap was performed by two Indian surgeons and then published in the Gentleman's Magazine of London [1]. The first nasal reconstruction using the Indian method in England was performed in 1814 and was published two years later in Germany. Von Graefe performed a complete nasal reconstruction in 1818 and illustrated the technique in his book, coining the term "plastic surgery." Warren was the first 
to complete a neo-rhinoplasty using the Indian method in the United States in 1837 [1-5]. Since then, many improvements have been described [6]. Unlike superficial defects, throughand-through nasal reconstruction presents the challenge of providing adequately vascularized lining and skin simultaneously [6-9]. Previous papers have described multi-staged procedures, second local flap harvesting, and free tissue transfer to provide lining to circumvent this problem [7]. In this paper, the authors

\section{Table 1. Patient data}

\begin{tabular}{|llclc|}
\hline $\begin{array}{l}\text { Age }(\mathrm{yr}) / \\
\text { Sex }\end{array}$ & \multicolumn{1}{c}{ Tumor type/Site } & TNM staging & Flap design & Follow-up \\
\hline 78/M & SCC/Alar and sidewall & $\mathrm{T}_{4} \mathrm{~N}_{0} \mathrm{M}_{0}$ & Wing-shaped & 22 \\
67/M & BCC/Dorsum and left sidewall & $\mathrm{T}_{3} \mathrm{~N}_{0} \mathrm{M}_{0}$ & Heart-shaped & 34 \\
58/M & SCC/Dorsum and tip & $\mathrm{T}_{4} \mathrm{~N}_{1} \mathrm{M}_{0}$ & Heart-shaped & 35 \\
03/F & SCC/Tip & $\mathrm{T}_{3} \mathrm{~N}_{0} \mathrm{M}_{0}$ & Heart-shaped & 43 \\
72/M & BCC/Alar and tip & $\mathrm{T}_{3} \mathrm{~N}_{0} \mathrm{M}_{0}$ & Wing-shaped & 56 \\
86/F & BCC/Alar & $\mathrm{T}_{3} \mathrm{~N}_{0} \mathrm{M}_{0}$ & Wing-shaped & 18 \\
59/M & SCC/Dorsum and tip & $\mathrm{T}_{4} \mathrm{~N}_{0} \mathrm{M}_{0}$ & Heart-shaped & 28 \\
\hline
\end{tabular}

Patients are numbered from 1 to 7 ; male $(\mathrm{M})$ and female $(\mathrm{F})$; SCC, squamous cell carcinoma; BCC, basal cell carcinoma; the tumor site was according to the subunit of contour; follow-up is expressed in months.

\section{Fig. 1. Bi-pedicled forehead flap design}

The flap is equipped with a rich vascular network deriving from the intersection of the supratrochlear and supraorbital vessels on the one hand and to the angular vessels on the other.

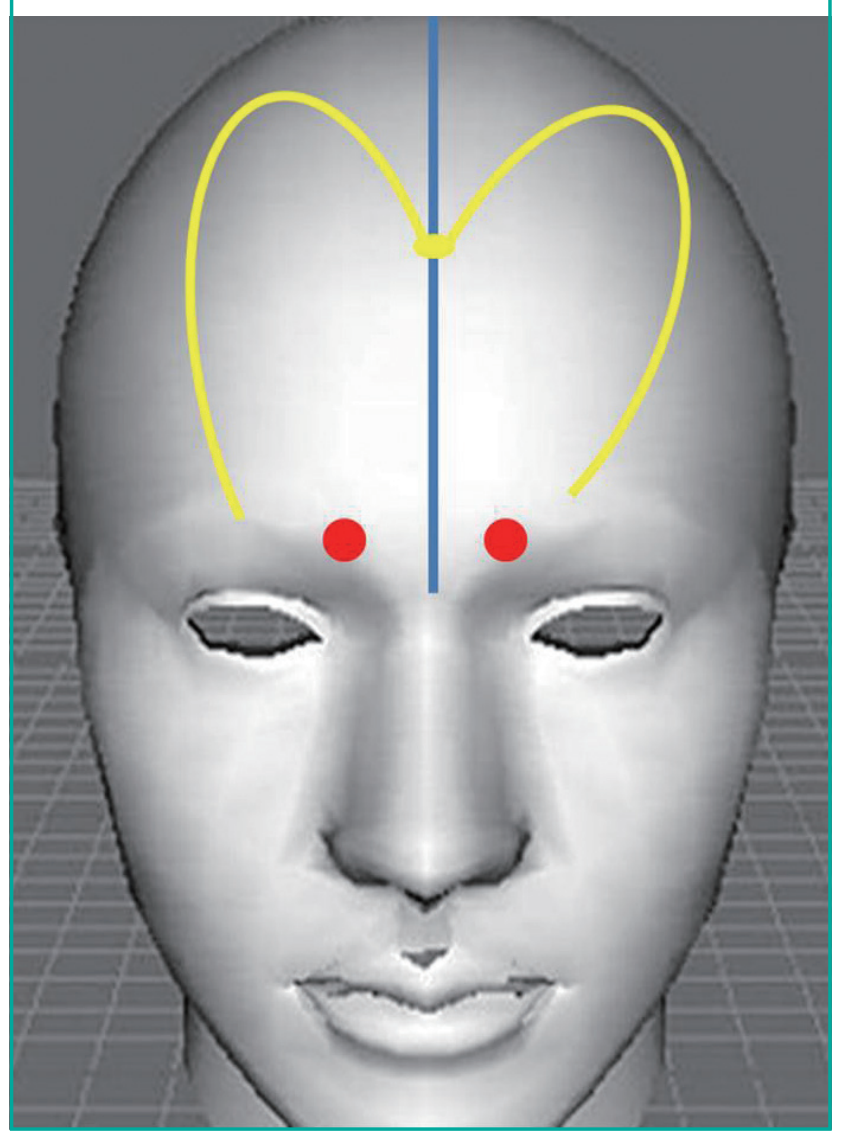

describe a modification of the forehead flap raised on both the supratrochlear vessels to reconstruct through-and-through nasal defects following non-melanoma skin cancer resection.

\section{METHODS}

Seven patients suffering non-melanoma skin cancer underwent neo-rhinoplasty with a bi-pedicled forehead flap. The patients were aged between 58 and 86 years, with a mean age of 63.4 years. All of the tumors were excised using traditional surgery, and reconstruction was performed simultaneously following pathological analysis by fresh frozen section. Surgery was performed under general anesthesia in 4 of the patients following evaluation by the anesthesiology department of our hospital. Tolerance of the surgical procedure was acceptable in all of the cases, and no anesthetic or surgical complications were detected. The patient details, tumor type and site, TNM staging, reconstruction method, and follow-up period are listed in Table 1. The flap was designed according to the defect, forehead conformation, and sex of the patient. One patient required a level 1 neck dissection because of lymph node involvement. In the remaining cases, lymphatic metastases were excluded clinically and were radiologically assessed both preoperatively and during follow-up.

\section{Surgical technique}

The pedicle of the flap is situated just over the glabella, between the eyebrows and based on the supratrochlear and angular vessels (Fig. 1) [2]. Use of vasoconstrictors is advised. The supratrochlear vessels are identified with a Doppler probe lateral to the medial frown crease. The lobes are usually raised in the frontal region but the dissection can be extended up to the temporal-parietal area in males who are bald at the temples. The flap is of full thickness near the pedicle and is thinner at the top of the lobes, at which point it is composed mainly of skin, the muscular layer, and the periosteum if necessary. The flap length is variable according to the position on the forehead: when central, the flap should be shorter. When extended to the temporoparietal area, the axis will be longer and will feature a major arch of rotation. Even if no specific roles exist for lobe harvesting procedures (the capillitium should never be included), the authors recommend that the base of the pedicle be 2 to $3 \mathrm{~cm}$ wide for a safer and more reliable flap [2]. Both the lobes are rotated on the defect: one is turned towards the nasal lining and the other is overlapped to restore the nasal skin. The lobes will remain in a sandwich fashion for 3 to 4 weeks to allow for vascular delay; thereafter, the glabellar pedicle can be resected and the remaining tissue can be attached to the forehead. The donor site 


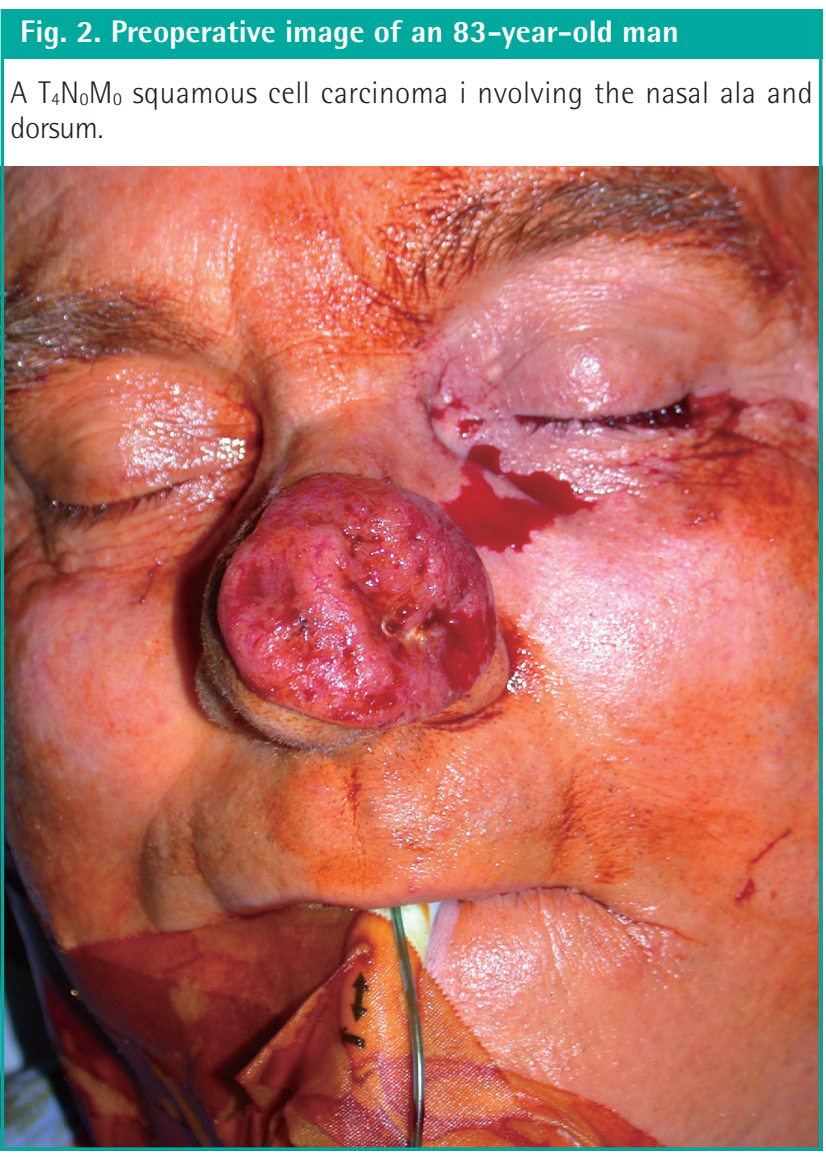

is usually covered by skin grafts. This flap is equipped with a rich vascular network thanks to the intersection of the supratrochlear and supraorbital arteries on the one hand and to the angular veins on the other.

\section{RESULTS}

In this series of seven patients, subtotal nasal reconstruction was achieved with a bi-pedicled forehead flap. Reconstruction was well accepted in all cases. The heart-shaped forehead flap was harvested in cases of subtotal nose involvement (Figs. 2-5), while minor defects or defects involving the nasal tip were reconstructed with a wing-shaped forehead flap (Figs. 6, 7). No cartilaginous or osseous support was necessary. The forehead represented the only donor site morbidity in all of the cases. A more demanding microsurgical approach was avoided consistent with the sex, age, and decision of the patients who were fully informed of the surgical options. Tumor-free margins were achieved in all cases and the results were confirmed by intraoperative frozen section evaluation. All of the patients were found to be free from disease upon follow-up. Two patients agreed to undergo further nasal reshaping.

\section{Fig. 3. Intraoperative results following reconstruction}

Intraoperative image showing the full-thickness defect of the nasa framework and the surgical planning of a bi-pedicled forehead flap.

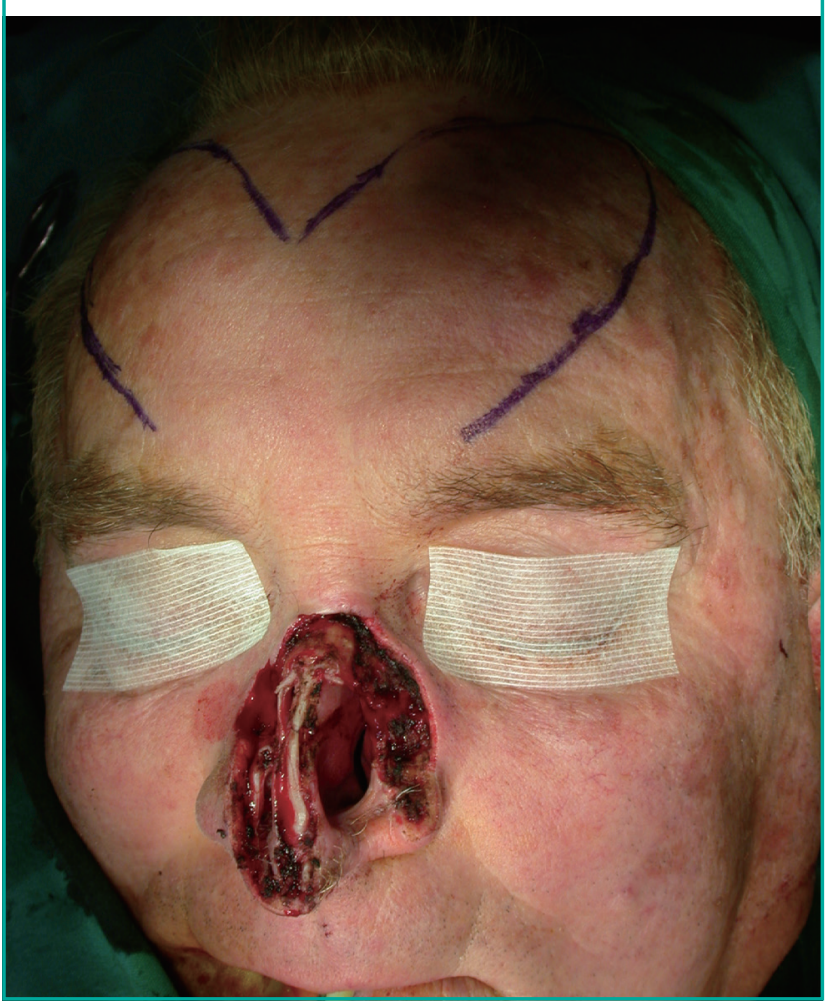

\section{DISCUSSION}

The management of nasal defects following surgery is influenced by several factors, including histology, location, staging, and previous treatment regimens employed [2-5]. These characteristics define the degree of tumor control and therefore the reconstruction [4]. The size and location of the defect as well as the availability of adjacent skin are further factors to be considered. The patients' age, co-morbidities, and aesthetic goals must also be included in the decision making process $[8,9]$. The ideal reconstruction closes the defect following tumor resection with a good tissue match and no stenosis or distortion. Immediate closure decreases morbidity, prevents hemorrhage and minimizes wound infection [10]. The forehead represents a maximum tissue reservoir for reconstructing large, fullthickness defects of the nose. The forehead flap is the method of choice for closure of nasal defects that are not amenable to more simple reconstructive options $[11,12]$. This flap is based upon the supratrochlear artery, which crosses the supero-medial orbit approximately 1.7 to $2.2 \mathrm{~cm}$ lateral to the midline, and courses vertically in a paramedian position approximately $2 \mathrm{~cm}$ lateral to the midline [3-9]. The Doppler location of the supratrochlear artery localizing its exact position allows flap harvesting with a 


\section{Fig. 4. Postoperative results after 10 days}

The folded flaps for reconstructing both the nasal ling and the skin during the time necessary to achieve the vascular delay can be seen.

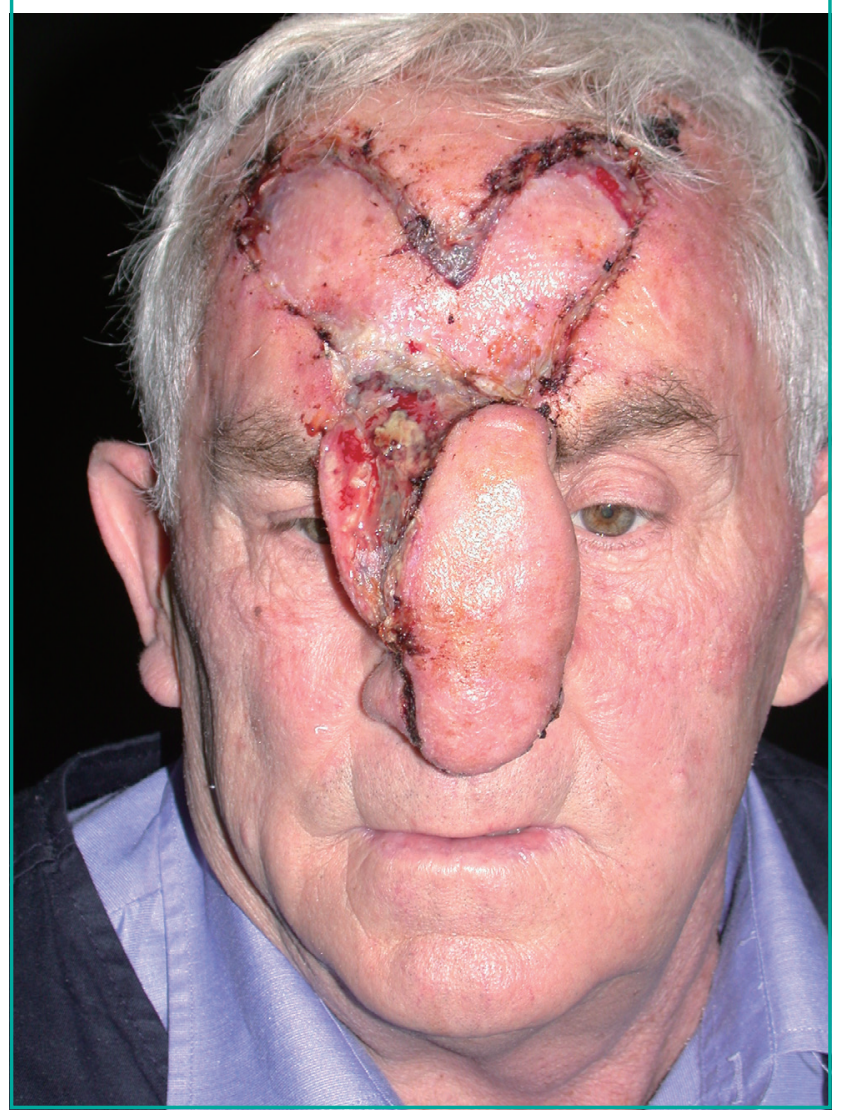

relatively narrow pedicle of less than $1.5 \mathrm{~cm}$. This facilitates pivot rotation providing a more effective flap length and preventing donor site deformity in the glabellar region [5]. Vascular delay is performed at three weeks with appropriate debulking and contouring at the recipient site [3]. If the lining is missing, its replacement should be the first consideration. Various alternatives can be considered to replace the internal nasal lining, including skin grafting, folding the distal aspect of the cutaneous flap on itself, intranasal pedicled mucosal flaps or epithelial turn-in flaps from around the defect. Skin grafts may contract and do not allow major cartilage replacement at the first stage. Small intranasal pedicled mucosal flaps (including septal and bipedicled septal/vestibular flaps) may be harvested ensuring an excellent blood supply. Nonetheless, a composite septal flap may not reach down to the nostril without compromising midline support and significant secondary donor site morbidity may lead to septal perforation formation [5-8]. On this basis, the revisited bilobed flap has an axial vascular pattern, which represents a valid alternative to reconstructing both the nasal lining and skin simultaneously with reduced complications. In particular, it is a double transposition flap, and its geometry enables a better

\section{Fig. 5. Postoperative frontal view after 3 years}

Full-thickness nasal defects was repaired using a bi-pedicled heartshaped forehead flap.

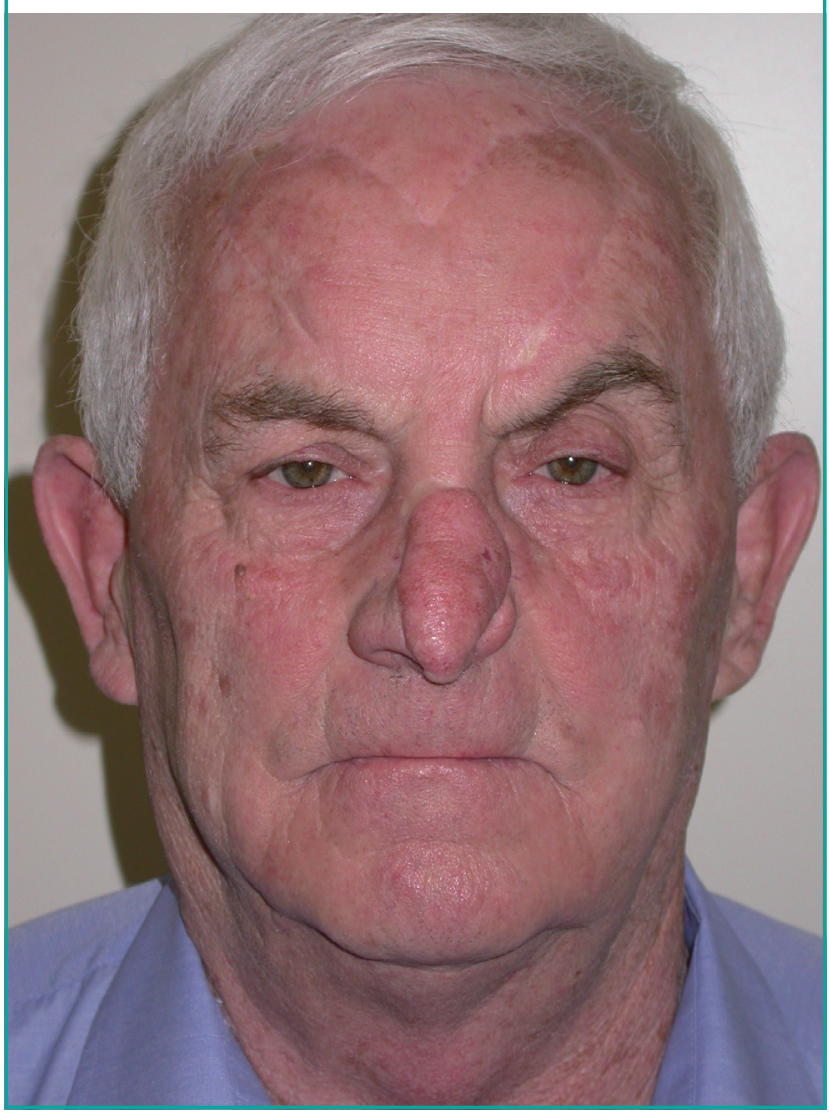

distribution of the tensile forces along its rotation axis, avoiding the distortions and redundancy generated by other flaps. Our bi-pedicled flap has two main differences, as described in the plastic surgery literature. First, the traditional bilobed flap is a double transposition flap with a single pedicle in which the first flap is transposed into the defect and the second smaller flap is transposed to repair the secondary defect resulting from the larger flap transposition. Our flap differs from the classical description since it is equipped with two distinct vascular axes and two transposition flaps of equal dimensions fitting the receiving site (Fig. 3). Second, a bilobed forehead flap was described more than 30 years ago by McGregor for restoring a large nasal skin defect without lining involvement resulting from tumor excision [12]. Our bi-pedicled forehead flap is used to reconstruct full-thickness defects by restoring the nasal lining with a transposition flap and the nasal skin by a contralateral specular flap. Consequently, this reconstructive technique is feasible for reconstructing full-thickness nasal defects of varying sizes of the whole nasal framework.

The bi-pedicled forehead flap is not suggested for the restoration of superficial defects but for total or subtotal nasal recon- 


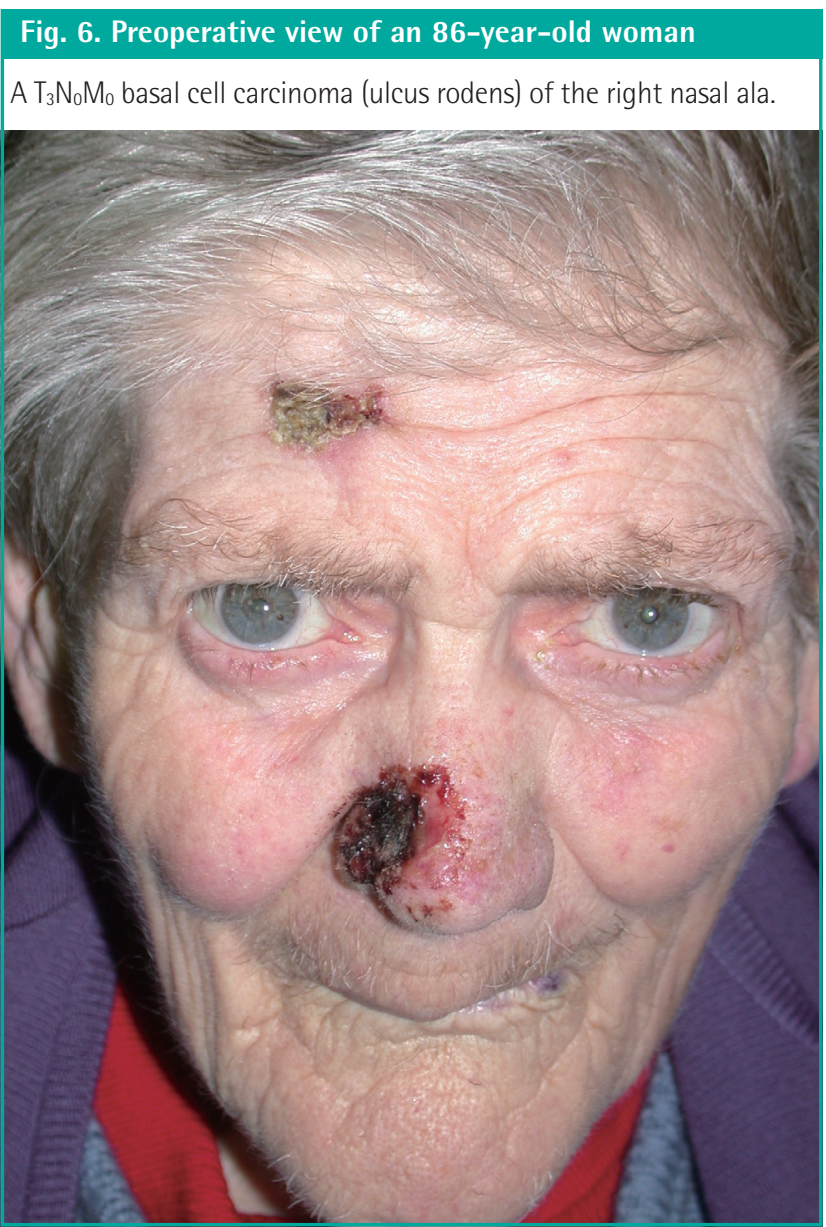

struction [1]. Indeed, the folded flap requires the use of bone or cartilage supports and includes simultaneous restoration of the nasal lining and skin. The forehead is usually well repaired following delay, with some exceptions, notably the superolateral areas, which remain covered by skin grafts. Nonetheless, the cosmetic outcome of the donor site is acceptable and well-tolerated $[8-10,13]$. The forehead is not interrupted with a vertical scar commonly associated with the traditional forehead flap but instead exhibits gentle and curvilinear incisions, except laterally. Further refinements can be performed postoperatively. Limitations include discomfort due to the vascular delay (3 weeks) and a possible revision to thin the folded flap. Additionally, even if a simultaneous reconstruction of the nasal lining and facial skin can be achieved with a median or paramedian forehead flap grafted on the deeper layer, the flap often lacks bulk in many cases and grafting of cartilaginous or osseous support almost always proves problematic $[11,13]$. Furthermore, free tissue transfer significantly increases the difficulty, operating time, cost, and bulk of the tissue transferred [11]. The bi-pedicled forehead flap, equipped with two thick and hairless lobes, can be considered a valuable resource and a valid alternative even for

\section{Fig. 7. Postoperative view after 11 months}

The full-thickness defect was repaired with a bi-pedicled wing-shaped forehead flap. The frontal basal cell carcinoma was extirpated and closed with direct suturing.

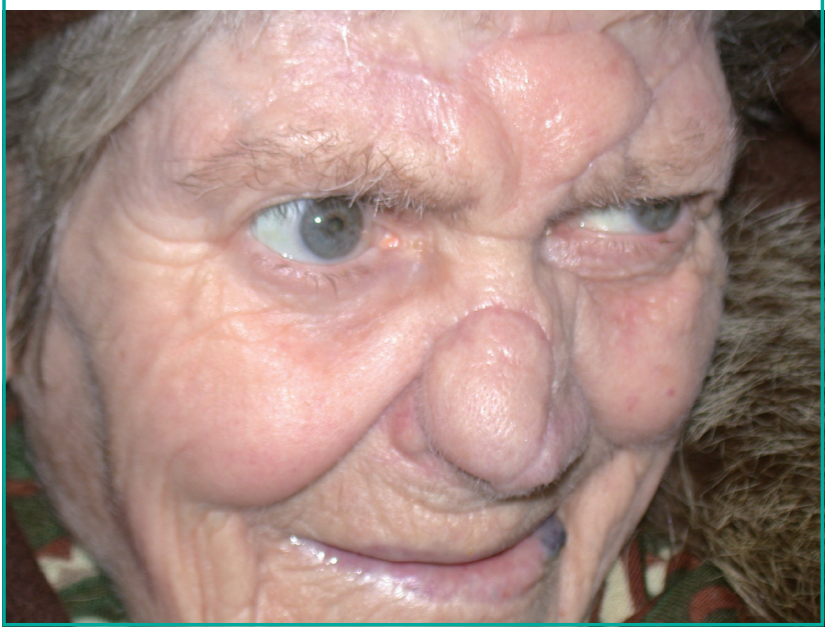

other complex facial defects in the orbital (exenteratio orbitae), zygomatic, and cheek regions.

\section{REFERENCES}

1. Zaoli G. La chirurgia ricostruttiva nei carcinomi del distretto cervico-facciale. In: Piccin, editor. Il naso. Padova: Piccin Editore; 1978. p.159-208.

2. Lo Russo D. Descrizione di un lembo frontale monopeduncolato-bilobato per la ricostruzione della piramide nasale: lembo ad “asso di cuori”. Riv Ital Chir Plastica 1980;12:97105.

3. Menick FJ. Nasal reconstruction with a forehead flap. Clin Plast Surg 2009;36:443-59.

4. Bhrany $\mathrm{AD}$. Complex nasal reconstruction: a case study: reconstruction of full-thickness nasal defect. Facial Plast Surg Clin North Am 2011;19:183-95.

5. Selcuk CT, Ozalp B, Durgun M, et al. Reconstruction of fullthickness nasal alar defects using cartilage-supported nonfolded nasolabial flaps. J Craniofac Surg 2012;23:1624-6.

6. Qian C, Yaodong X, Xiaoming H, et al. Repair of full-thickness alar defects. Dermatol Surg 2012;38:1639-44.

7. Wentzell JM. Dorsal nasal flap for reconstruction of fullthickness defects of the nose. Dermatol Surg 2010;36:11718.

8. Masic T, Lincender I, Dizdarevic D. Reconstruction of total and subtotal nose defects. Med Arh 2010;64:110-2.

9. Garces JR, Guedes A, Alegre M, et al. Bilobed flap for fullthickness nasal defect: a common flap for an uncommon indication. Dermatol Surg 2009;35:1385-8. 
10. Bickle K, Bennett RG. Combined hinge flap full-thickness skin graft for a through-and-through nasal defect. Dermatol Surg 2008;34:389-92.

11. Potter JK, Ducic Y, Ellis E 3rd. Extended bilaminar forehead flap with cantilevered bone grafts for reconstruction of fullthickness nasal defects. J Oral Maxillofac Surg 2005;63:56670.
12. McGregor JC, McLean NR. Reconstruction of a large nasal defect using a bilobed forehead flap. Ann Plast Surg 1982; 9:419-24.

13. Ayhan M, Aytug Z, Karatas-Silistreli O, et al. The use of nail enfolded flaps for reconstruction of full-thickness ala nasi defects: an early report of experience. Acta Chir Plast 2006; 48:79-84. 